\title{
Modelling of the response of acoustic piezoelectric resonators in biosensor applications - Part 1: The general theoretical analysis
}

\author{
M. V. Voinova ${ }^{1,2, *}$ \\ ${ }^{1}$ Chalmers University of Technology, Gothenburg, Sweden \\ ${ }^{2}$ National Technical University, KhPI, Kharkov, Ukraine \\ *now at: Chalmers University of Technology, Gothenburg, Sweden \\ Correspondence to: M. V. Voinova (marina.voinova@chalmers.se)
}

Received: 30 August 2014 - Revised: 17 November 2014 - Accepted: 2 December 2014 - Published: 7 April 2015

\begin{abstract}
Acoustic piezoelectric resonators are widely used as precise analytical chemistry tools for the realtime monitoring of a negligibly small amount of surface-attached mass of biological components, in particular, in environmental biosensor measurements. The surface acoustic wave (SAW)-based sensors and the quartz crystal microbalance (QCM) compared in our work belong to the leading group due to their considerable advantages. These piezoelectric resonators are considered now as high-resolution analytical tools allowing researchers to discriminate between components due to the selective polymer coating on the resonator surface. The gravimetrical measurements performed with the SAW-based or QCM sensors provide the experimental data with high precision for the detection of surface mass for the thin adsorbed layer rigidly attached to the oscillator surface. The new challenge is the analysis of soft and biological materials, where the viscous losses of energy can essentially influence measured characteristics. Modelling is the important part of the analysis allowing researchers to quantify the results of the experiments. The present work provides a general theory of SH-SAW devices probing soft and biological materials. The results are compared with QCM-D operated in liquid media.
\end{abstract}

\section{Introduction}

Two types of devices are accepted as acoustical sensors now: SAW (surface acoustic wave)-based and BAW (bulk acoustic wave)-based resonators. The quartz crystal microbalance (QCM) sensors (BAW resonators), originally used for the gravimetric measurements in gases mostly (Sauerbrey, 1959), and later in viscous Newtonian liquids (Kanazawa and Gordon, 1985; Kanazawa et al., 2008; Rodahl and Kasemo, 1996; Rodahl et al., 1995, 1997), are widely used now for viscous/viscoelastic films studies (Johannsmann et al., 1992; Johannsmann, 2007, 2015; Lucklum and Hauptmann, 2000, 2006), as well as for biosensor purposes (Janshoff and Steinem, 2001; Marx, 2003; Rodahl et al., 1997). Surface acoustic wave sensors have a number of advantages in comparison with the QCM. First of all, it is the high resonance frequency $(100 \mathrm{MHz}-\mathrm{a}$ few $\mathrm{GHz}$ frequencies operation region, while typically, $3-10 \mathrm{MHz}$ one for the QCM). Surface acoustic wave resonators are commonly used in the air and gases (Gaso et al., 2013; Kalantar-Zadeh et al., 2001, 2003a, b, 2006; Kovacs and Venema, 1992; Länge et al., 2008; Penza et al., 1998). Starting from the original research in Love wave devices (Love, 1911) rapid development in SAW gas sensors was made due to appearance of new advanced materials for the sensors' surface coating (Penza et al., 2004, 2007, 2009). In spite of the experimental problems in the late 1980s and early 1990s, in a number of original works (Gizeli et al., 1992a, b, 1996, 1997) it was shown that SAW devices with horizontal wave polarization (SH SAWs) can be used for measurements in liquids, in particular, as viscosity sensors (Ricco and Martin, 1987; Kalantar-Zadeh et al., 2003; Powell et al., 2004). This finding opens a way for the biosensor applications since water is an essential component for the proteins, nucleic acids and other biological molecules. The specific chemical treatment of the resonator surface permits the highly selective detection of biological components in a 
water solution, enabling the SH-SAW devices for the in situ monitoring of protein adsorption, bacteria toxins, DNA studies and immunological analysis (Freudenberg et al., 2001; Kalantar-Zadeh et al., 2003a, b; Länge et al., 2008; Rapp et al., 2007). Another engineering trend for the life science applications is a combination of SAW sensors with the peripheral microfluidics which is an excellent perspective, e.g. for the lab-on-a-chip and immunological setup construction (Länge et al., 2008; Melzak et al., 2007). Both methods - the QCM and SH-SAW resonators - are modern precise bioanalytical tools and both of them demonstrate beneficial features. The dynamics of a film immersed into a bulk Newtonian liquid and firmly attached to the oscillating crystal substrate can be studied with the help of acoustic resonators by using several piezomaterials with different acoustical characteristics. For the quartz, different cut of the crystal allows the excitation of either surface acoustic waves or bulk acoustic waves. For example, the AT-cut quartz plate is typically used for the quartz crystal microbalance resonators.

The theoretical modelling is important for the quantitative analysis and interpretation of the experimental results. Many efforts have been reported in this area since the 1990s (Gulyaev and Plessky, 1997; Gulyaev, 1998; Kalantar-Zadeh et al., 2003a, b; Kosevich et al., 1995; Newton et al., 2001; Powell et al., 2004; Kovacs and Venema, 1992; McHale et al., 2001; Plessky, 1995). The challenge is to include the softness (or viscoelasticity) of the testing material into consideration, specifically, when the resonator operates in a liquid. To our knowledge, this problem has not been solved analytically.

In the present paper, the viscoelastic correction was found for the characteristics of surface acoustic waves with horizontal polarization. The rigorous mathematical expressions for the shift in the phase wave velocity and attenuation (the dissipation coefficient) for this type of SAWs are derived for the first time. The results of the theory are compared with the "missing mass" effect predicted earlier (Voinova et al., 1999, 2002) for the BAWs (specifically important for QCM-D, a device which allows researchers the simultaneous measurements of the resonance frequency and the dissipation in liquid applications; Rodahl and Kasemo, 1996; Rodahl et al., 1995, 1997).

\section{The SAW and BAW resonators - the dynamics of viscoelastic films in liquids}

We study surface acoustic waves with horizontal polarization (SH waves) propagating in the two-layer system. In the paper we employed our method developed earlier in Kosevich et al. (1995) and Voinova et al. (1997) and generalized the theory for the case of a viscoelastic thin layer under a bulk liquid (water solution, for example). Such a system has been analysed in details in our earlier works (Voinova et al., 1999, 2002; Voinova, 2009) for the BAWs excited in the quartz crystal microbalance (QCM). In the last section of the paper we compare the QCM results and our analytical results ob- tained for the surface acoustic waves. The importance of this research is the analytical formulae obtained for the shift in the phase velocity and the attenuation of acoustic SH waves which can be measured experimentally and, thus, verified for the coatings with known properties (for example, supported membranes or polymer films). In the air, the surface acoustic wave phase velocity change due to the presence of rigid two-layer film of different densities is given by the following expression (Voinova et al., 1997):

$$
\frac{\Delta V}{V_{0}}=\frac{1}{2} \frac{\omega^{2} \rho_{1}^{2} h^{2}}{V_{0}^{2} \rho_{0}^{2}}\left\{1+\frac{\rho_{2}}{\rho_{1}}\right\}^{2},
$$

where

$V_{0}=\sqrt{g_{0} / \rho_{0}}=$ const

is the (constant) wave velocity in the solid oscillating substrate of density $\rho_{0}$ and of shear elastic modulus $g_{0}$. This expression corresponds to the Love wave type: the shift in the phase velocity is a quadratic function of the frequency $\omega=2 \pi f_{0}$ and the surface film mass $m_{\mathrm{S}}=\rho h$.

The dependence of the phase velocity on the film surface mass makes it possible to use Love wave devices for the gravimetrical measurements. For bulk acoustic waves, the measured shift in the resonance frequency is proportional to the surface mass and the frequency. The linear relationship between the shift in the resonance frequency and the surface mass which causes this shift (the principle of microbalance for the QCM devices) is given by the so-called Sauerbrey relation (Sauerbrey, 1959):

$$
\frac{\Delta f}{f_{0}}=\frac{\rho h}{m_{0}} \text {. }
$$

The correction due to the viscous or viscoelastic properties of the film material may essentially influence the results. For the SH-SAW case, for the thin film of shear viscosity $\eta$, we get

$$
\frac{\Delta V}{V_{0}}=\frac{1}{2} \frac{\omega^{2} \rho_{1}^{2} h^{2}}{V_{0}^{2} \rho_{0}^{2}}\left\{1-\left(\frac{\omega \eta}{\rho V_{0}^{2}}\right)^{2}\right\} .
$$

Below it is shown that when the viscoelastic film is covered with the bulk liquid on the top, the viscous corrections appeared already in the linear terms on the film thickness. This "missing mass" effect has been predicted first for the BAW resonators (QCM or QCM-D in liquids) (Voinova et al., 1999, 2002):

$$
\frac{\Delta f}{f_{0}}=\frac{\rho h}{m_{0}}(1-\alpha),
$$

where $\alpha$ is a function of the viscosity and elasticity of the coating material.

In the next section the theoretical formulation of the model is presented for the SH-SAW propagated into a layered system onto the surface of a solid substrate. The similar physical 


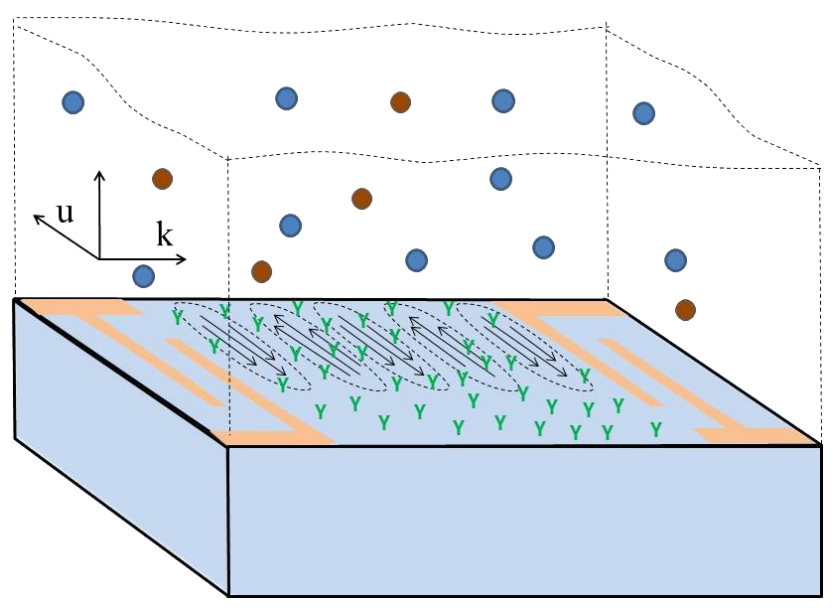

Figure 1. Sketch of the two-layer system deposited onto the surface of SH-surface acoustic resonator: the first layer is a supported membrane; the second is the bulk aqueous solution.

phenomenon of the "missing mass" found in the calculated shift of the phase velocity of the resonator when the upper layer is a viscous bulk liquid.

\section{Formulation of the theoretical model}

We analyse the surface acoustic waves with horizontal polarization propagating in the two-layer system with the geometry depicted on Fig. 1. The viscoelastic properties of the surface-adjacent thin film are described with the complex shear modulus of elasticity

$g^{*}=g^{\prime}+i g^{\prime \prime}$,

where the real part is the storage modulus, $g^{\prime}$, and the imaginary part is the loss modulus, $g^{\prime \prime}$, respectively. The top bulk layer is assumed to be Newtonian viscous liquid which is characterized with the shear viscosity $\eta$.

The displacement of the solid substrate boundary is given by

$u_{y}=u_{0} \exp \left(\kappa^{2} x-i k z\right) \exp (i \omega t)$

where $\kappa=\sqrt{k^{2}-\frac{\omega^{2}}{V_{0}^{2}}}$ is the inverse penetration depth of the acoustic surface $\mathrm{SH}$ wave into the solid support. The substrate motion is described by the equation

$\rho_{0} \ddot{u}_{y}=g_{0}\left(\frac{\partial^{2} u_{y}}{\partial z^{2}}+\frac{\partial^{2} u_{y}}{\partial x^{2}}\right)$.

Assuming the validity of the absence of the interfacial slippage, the standard boundary conditions are as follows:

$$
\begin{gathered}
\text { At } z=0, \\
v_{y}=\frac{\partial u_{y}(x, z, t)}{\partial t}, \\
\sigma_{z y}=g^{*}\left(\frac{\partial u_{y}}{\partial z}\right), \\
\sigma_{z y}=g_{0}\left(\frac{\partial u_{y}}{\partial z}\right) .
\end{gathered}
$$

At the film liquid interface $(z=h)$,

$n_{k} \sigma_{i k}^{(1)}=n_{k} \sigma_{i k}^{(2)}$

The interface (air-liquid) at $z=H$ is considered as a free surface, and the surface film thickness $h \ll H$.

Equations (1) and (2) together with the boundary conditions (3)-(6) lead to the dispersion equation which is presented and analysed in the next section.

\subsection{The dispersion equation}

The dispersion equation in the long-wave approximation is given by the expression

$\kappa^{2}=\frac{g^{* 2} \zeta_{1}^{2}}{g_{0}^{2}}\left\{\frac{\left(g^{*} \zeta_{1}-G^{*} \zeta_{2}\right)-e^{2 \zeta_{1} h}\left(g^{*} \zeta_{1}+G^{*} \zeta_{2}\right)}{\left(g^{*} \zeta_{1}-G^{*} \zeta_{2}\right)+e^{2 \zeta_{1} h}\left(g^{*} \zeta_{1}+G^{*} \zeta_{2}\right)}\right\}^{2}$,

where

$$
\begin{aligned}
\zeta_{1} & =\sqrt{k^{2}-\frac{\rho_{1} \omega^{2}}{g^{*}}}, \\
\zeta_{2} & =\sqrt{k^{2}-\frac{\rho_{2} \omega^{2}}{G^{*}}}, \\
g^{*} & =g^{\prime}+i g^{\prime \prime}, \\
G^{*} & =G^{\prime}+i G^{\prime \prime} .
\end{aligned}
$$

Here we introduce the complex shear viscoelastic moduli for the film $\left(g^{*}\right)$ and for the bulk layer $\left(G^{*}\right)$, indices "1" and " 2 " correspond to the first layer material parameters and the top liquid, respectively. In case of Newtonian liquid of shear viscosity $\eta$, the latter parameter reduces to the $G^{*}=i \eta_{2} \omega$ value.

From the analysis of the dispersion relation (7) in the long wavelength approximation, for the acoustically thin film and for $h \ll H$, in the linear approximation on film thickness one 
can find for the phase velocity shift

$\Delta V / V_{0} \approx$

$$
\frac{\omega^{3 / 2} \rho_{1} h_{1} V_{0}^{2}}{2 g_{0}^{2}} \sqrt{2 \eta_{2} \rho_{2}}\left\{1-\frac{\eta_{2} \omega\left(g^{\prime}+g^{\prime \prime}\right)}{g^{\prime 2}+g^{\prime \prime 2}} \frac{\rho_{2}}{\rho_{1}}\right\}
$$

and for the attenuation of surface acoustic waves

$\Gamma \approx$

$$
\frac{\omega^{2}}{2 g_{0}^{2}} V_{0}\left\{\eta_{2} \rho_{2}+h_{1} \rho_{1} \sqrt{2 \omega \eta_{2} \rho_{2}}\left[1+\frac{\rho_{2}}{\rho_{1}} \frac{\eta_{2} \omega\left(g^{\prime}-g^{\prime \prime}\right)}{\left(g^{\prime 2}+g^{\prime \prime 2}\right)}\right]\right\} .
$$

In case of two pure viscous layers of immiscible liquids with different viscosities and densities (indices 1 and 2, respectively) one can easily get

$\Delta V / V_{0} \approx \frac{\omega^{3 / 2} \rho_{1} h_{1} V_{0}^{2}}{2 g_{0}^{2}} \sqrt{2 \eta_{2} \rho_{2}}\left(1-\frac{\rho_{2}}{\rho_{1}} \frac{\eta_{2}}{\eta_{1}}\right)$

$\Gamma \approx \frac{\omega^{2}}{2 g_{0}^{2}} V_{0}\left\{\rho_{2} \eta_{2}+h_{1} \rho_{1} \sqrt{2 \rho_{2} \eta_{2} \omega}\left[1-\frac{\rho_{2}}{\rho_{1}} \frac{\eta_{2}}{\eta_{1}}\right]\right\}$.

These formulae (Eqs. 10, 11) can describe, for example, a lipid bilayer membrane covered with the aqueous solution (since the bilayer shows no resistance for in-plane shear deformation and thus behaves as a 2-D liquid) or some other immiscible liquid layers.

In the contrast, for the pure elastic thin film considering "dry conditions" (for example, when the resonator operated in gaseous environment) it was found (Kosevich et al., 1995) that the phase velocity shift is proportional to the square of the surface film mass and frequency:

$\Delta V / V_{0} \approx \frac{\omega^{2} \rho_{1}^{2} h_{1}^{2} V_{0}^{2}}{2 g_{0}^{2}}\left(1-\frac{\rho_{0}}{\rho_{1}} \frac{g^{\prime}}{g_{0}}\right)^{2}$.

This is in agreement with the result reported in McHale et al. (2002). The analysis shows that the thin rigid (or pure elastic) surface-adjacent film is acting as a Love wave guide (under assumption that the substrate is infinite).

\section{BAW resonators: the QCM-D results}

To compare the results derived here for the surface acoustic waves with the horizontal polarization, we also present the analytical expressions for the characteristics of QCM-D, namely, for the resonance frequency shift, $\Delta f$, and the dissipation factor, $\Delta D$, obtained in our earlier works (Voinova et al., 1999, 2002). For the acoustically thin film under a bulk water, we obtained the following expressions:

$\Delta f \approx-\frac{\eta_{\mathrm{L}}}{2 \pi m_{0} \delta_{\mathrm{L}}}-\frac{h_{1} \rho_{1} \omega}{2 \pi m_{0}}\left\{1-\frac{2}{\rho_{1}}\left(\frac{\eta_{\mathrm{L}}}{\delta_{\mathrm{L}}}\right)^{2} J^{\prime \prime}\right\}$,

$$
\begin{aligned}
\Delta D & \approx \frac{1}{\pi f m_{0}}\left\{\frac{\eta_{\mathrm{L}}}{\delta_{\mathrm{L}}}+2\left(\frac{\eta_{\mathrm{L}}}{\delta_{\mathrm{L}}}\right)^{2} h_{1} \omega J^{\prime}\right\}, \\
\delta_{\mathrm{L}} & =\sqrt{2 \eta_{\mathrm{L}} / \rho_{\mathrm{L}} \omega}, \\
J^{\prime} & =\frac{g_{1}^{\prime}}{g_{1}^{\prime 2}+g_{1}^{\prime \prime 2}}, \\
J^{\prime \prime} & =\frac{g_{1}^{\prime \prime}}{g_{1}^{\prime 2}+g_{1}^{\prime \prime 2}} .
\end{aligned}
$$

Here indices " 1 " and " $L$ " denote a thin film and bulk liquid, respectively, $\delta_{\mathrm{L}}$ is the viscous penetration depth, i.e. the distance over which the transverse wave amplitude falls off by a factor of $e, J^{\prime}$ and $J^{\prime \prime}$ is the compliance of the viscoelastic film (i.e. the ratio of the storage and loss components). Note that the expression (13) for the resonance frequency shift consists of one bulk term and two terms proportional to the film surface mass. The latter are opposite in sign: this finding of the mass correction is due to the presence of viscous liquid on the top of the film (the so-called "missing mass effect"; Voinova et al., 2002).

The expressions (8) and (9) presented in this work reveal the "missing mass" effect for the SH-SAW case, i.e. show the necessity of the correction of surface mass deduced from SAW device measurements in liquids.

\section{Conclusions}

Considering the results $($ Eqs. 8, 9) of the present paper, one can deduce that, in the case of surface acoustic wave propagation in two-layer geometry when the bulk layer is a viscous Newtonian liquid, calculation has shown that the phase velocity is proportional to the first order to the surface mass while in the air only to the square value (we would like to point out here that the calculations were performed in the approximation when the film thickness is taken as a small parameter).

Formulae (8) and (9), reporting the "viscous correction" of characteristics in the present paper, can provide valuable theoretical support for the liquid phase measurements using the surface acoustic wave sensors. The detailed analysis of the "missing mass" effect will be given in the second part of this work (Voinova and Vikström, 2015).

Acknowledgements. The author thanks Chalmers University of Technology for continuing support.

Edited by: Y. Li

Reviewed by: two anonymous referees 


\section{References}

Freudenberg, J., von Schickfus, M., and Hunklinger, S.: A SAW immunosensor for operation in liquid using a $\mathrm{SiO} 2$ protective layer, Sensor. Actuat. B-Chem., 76, 147-151, 2001.

Gaso, M. M. I. R., Jiménez, Y., Francis, L. A., and Arnau, A.: Lovewave biosensors: a review, in: State of the Art in Biosensors - General Aspects, edited by: Rinken, T., chapter 11, INTEC, 2013.

Gizeli, E., Goddard, N. J., and Lowe, C. W.: A Love plate biosensor utilizing a polymer layer, Sensor. Actuat. B-Chem., 6, 131-137, 1992a.

Gizeli, E., Stevenson, A. C., Goddard, J., and Lowe, C. R.: A novel Love-plate acoustic sensor utilizing polymer overlayers, IEEE Trans. Ultrason. Ferr., 39, 657-659, 1992b.

Gizeli, E., Liley, M., Lowe, C. R., and Vogel, H.: Detection of supported lipid bilayers with the acoustic Love waveguide device: application to biosensors, Sensor. Actuat. B-Chem., 34, 295-300, 1996.

Gizeli, E., Mehta, H., and Lowe, C. R.: Calibration of the Love wave sensor utilizing phospholipid bilayers, Chem. Biol. Sensors and Analyt. Electrochem. Methods Proc., 97 (19) Symposium of Electrochemical Society and International Society of Electrochemistry, Paris, 1997.

Gulyaev, Yu. V.: Review of shear surface acoustic waves in solids, IEEE Trans. Ultrason. Ferroel. Freq. Control, 45, 935-938, 1998.

Gulyaev, Yu. V. and Plesski, V. P.: "Slow" acoustic surface waves in solids, Pis'ma v Zh. Tehn. Fiz., 3, 220-223, 1977.

Janshoff, A. and Steinem, C.: Quartz crystal microbalance for bioanalytical applications, in: Sensors update, edited by: Baltes, H., Hesse, J., and Korvink, J. G., Vol. 9, Wiley-VCH, Weinheim, 2001.

Johannsmann, D.: Studies of contact mechanics with the QCM, in: Piezoelectric Sensors, edited by: Steinem, C. and Janshoff, A., Vol. 5, Springer-Verlag, New York, 49-109, 2007.

Johannsmann, D.: Quartz crystal microbalance in soft matter research, Fundamentals and modeling, Springer, 398, 2015.

Johannsmann, D., Mathauer, T., Wegner, G., and Knoll, W.: Viscoelastic properties of thin films probed with a quartz-crystal resonator, Phys. Rev. B, 46, 7808, doi:10.1103/PhysRevB.46.7808, 1992.

Kalantar-Zadeh, K., Trinchi, A., Wlodarski, W., and Holland, A.: A novel Love-mode device based on a ZnO/ST-cut quartz crystal structure for sensing applications, Sensor. Actuat. B-Chem., 100, 135-143, 2001

Kalantar-Zadeh, K., Powell, D. A., Wlodarski, W., Ippolito, S., and Galatsis, K.: Comparison of layered based SAW sensors, Sensor. Actuat. B-Chem., 91, 303-308, 2003a.

Kalantar-Zadeh, K., Wlodarski, W., Chen, Y. Y., Fry, B. N., and Galatsis, K.: Novel Love mode surface acoustic wave based immunosensors, Sensor. Actuat. B-Chem., 91, 143-147, 2003b.

Kalantar-Zadeh, K., Powell, D. A., Sadek, A. Z., Wlodarski, W., Yang, Q. B., and Li, Y. X.: Comparison of $\mathrm{ZnO} / 64^{\circ} \mathrm{LiNbO} 3$ and $\mathrm{ZnO} / 36^{\circ} \mathrm{LiTaO} 3$ surface acoustic wave devices for sensing applications, Sensor Lett., 4, 135-138, 2006.

Kanazawa, K. and Gordon, J. G.: The oscillation frequency of a quartz crystal resonator in contact with a liquid, Anal. Chim. Acta, 175, 99-105, 1985.
Kanazawa, K., Frank, C. W., and Hardesty, J.: Resonances of soft films under liquids on the QCM, ECS Transactions, 16, 419-429, 2008.

Kosevich, A. M., Syrkin, E. S., and Voinova, M. V.: Acoustical sensors using Langmuir-Blodgett films, in: Acoustical Imaging Vol. 21, edited by: Jones, J. P., New York: Plenum Press, 191200, 1995.

Kovacs, G. and Venema, A.: Theoretical comparison of sensitivities of acoustic shear wave modes for (bio)chemical sensing in liquids, Appl. Phys. Lett., 61, 639-659, 1992.

Länge, K., Rapp, B. E., and Rapp, M.: Surface acoustic wave biosensors: a review, Anal. Bioanal. Chem., 391, 1509-1519, 2008.

Love, A. E. H.: Some problems of geodynamics, Cambridge University press, Cambridge, 1911.

Lucklum, R. and Hauptmann P.: The quartz crystal microbalance: mass sensitivity, viscoelasticity and acoustic amplification, Sensor. Actuat. B-Chem., 70, 30-36, 2000.

Lucklum, R. and Hauptmann P.: Acoustic microsensors - the challenge behind microgravimetry, Anal. Bioanal. Chem., 384, 667682, 2006.

Marx, K. A.: Quartz crystal microbalance: a useful tool for studying thin polymer films and complex biomolecular systems at the solution-surface interface, Biomacromolecules, 4, 1099-1120, 2003.

McHale, G., Newton, M. I., Martin, F., Melzak, K., and Gizeli, E.: Resonant conditions for Love wave guiding layer thickness, Appl. Phys. Lett., 79, 3542-3543, 2001.

McHale, G., Newton, M. I., and Martin, F.: Layer guided shear horizontally polarized acoustic plate modes, J. Appl. Phys., 91, 5735-5744, 2002.

Melzak, K. and Gizeli, E.: Love wave biosensors, in: Handbook of Biosensors and Biochips, edited by: Lowe, C. R., Cullen, D., Weetall, H. W., and Karube, I., John Wiley \& Sons, 2007.

Newton, M. I., Martin, F., Melzak, K., Gizeli, E., and McHale, G.: Harmonic Love devices for biosensing applications, Electron. Lett., 37, 340-341, 2001.

Penza, M., Mikella, E., and Anisimkin, V. I.: Monitoring of NH3 gas by LB polypyrole based SAW sensor, Sensor. Actuat. B-Chem., 47, 218-224, 1998.

Penza, M., Antolini, F., Vittori, M., and Antisari, M.: Carbon nanotubes as SAW chemical sensors materials, Sensor. Actuat. BChem., 100, 47-59, 2004.

Penza, M., Tagliente, M. A., Aversa, P., Re, M., and Cassano, G.: The effect of purification of single-walled carbon nanotube bundles on the alcohol sensitivity of nanocomposite LangmuirBlodgett films for SAW sensing applications, Nanotechnology, 18, 185502-185514, 2007.

Penza, M., Sberveglieri, G., Wlodarski, W., and Li, Y.: Nanomaterials for chemical sensing technologies, J. Sensors, 2009, 924941, doi:10.1155/2009/924941, 2009.

Plessky, V.: Surface acoustic waves in inhomogeneous media, Springer Verlag, Berlin, 1995.

Powell, D. A., Kalantar-Zadeh, K., and Wlodarski, W.: Numerical calculation of SAW sensitivity: application to $\mathrm{ZnO}-\mathrm{LiTaO} 3$ transducers, Sensor. Actuat. A-Phys., 115, 456-461, 2004.

Rapp, B., Länge, K., Rapp, M., and Guber, A.: Surface acoustic wave biosensors for biomedical applications, in: Biomedizinische Technik (BMT), Aachen, Germany, 26-29 September, 2007. 
Ricco, A. J. and Martin, S. J.: Acoustic wave viscosity sensor, Appl. Phys. Lett., 50, 1474-1476, 1987.

Rodahl, M. and Kasemo, B.: On measurement of thin overlayers with the quartz crystal microbalance, Sensor. Actuat. A-Phys., 54, 448-456, 1996.

Rodahl, M., Höök, F., Krozer, A., Brzezinski, P., and Kasemo, B.: A quartz crystal microbalance setup for frequency and Q-factor measurements in gaseous and liquid environments, Rev. Sci. Instrum., 66, 3924-3930, 1995.

Rodahl, M., Höök, F., Fredriksson, C., Keller, C. A., Krozer, A., Brzezinski, P., Voinova, M. V., and Kasemo, B.: Simultaneous frequency and dissipation factor QCM measurements of biomolecular adsorption and cell adhesion, Faraday Discuss., 107, 229-249, 1997.

Sauerbrey, G.: Verwendung von Schwingquarzen zur Wägung dünner Schichten und zur Mikrowägung, Z. Physik, 155, 206-212, 1959.
Voinova, M. V.: On mass loading and dissipation measured with acoustic wave sensors: A review, J. Sensors, 2009, 943125, doi:10.1155/2009/943125, 2009.

Voinova, M. and Vikström, A.: Modelling of the response of acoustic piezoelectric resonators in biosensors applications - Part 2: The "missing mass" effect, in preparation, 2015.

Voinova, M. V, Jonson, J., and Kasemo, B.: Dynamics of viscous amphiphilic films supported by elastic solid substrates, J. Phys. Chem. C, 9, 7799-7808, 1997.

Voinova, M. V., Rodahl, M., Jonson, M., and Kasemo, B.: Viscoelastic acoustic response of layered polymer films at fluid-solid interfaces: continuum mechanics approach, Phys. Scripta, 759, 391-396, 1999.

Voinova, M. V., Jonson, M., and Kasemo, B.: "Missing mass" effect in biosensors QCM applications, Biosens. Bioelectron., 17, 835841, 2002. 